\title{
Phonemic Variation of Urdu Loanwords in Standard British English
}

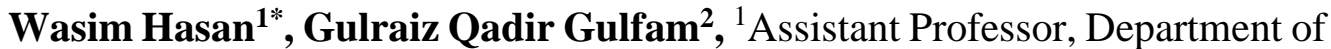 \\ English, National University of Modern Languages (NUML), Lahore Campus, \\ Pakistan, ${ }^{2}$ Lecturer, Department of English, National University of Modern \\ Languages (NUML), Lahore Campus, Pakistan, ${ }^{*}$ Corresponding Author, Email: \\ 1*whasan@numl.edu.pk
}

Borrowing is one of the most common sources of new words in languages and loanwords are one of the types of borrowings across language boundaries. The English language has adopted many words from other languages throughout history. In this process, loanwords sometimes undergo certain variations. The present study is about phonemic variation of Urdu loanwords in standard British English. A list of 135 Urdu words of daily use that English has borrowed was compiled purposively from Oxford Advanced Learners' Dictionary, 10th ed. (2020) for analysis. All words in the list were transcribed into IPA. Urdu pronunciation was checked from ("Urdu Lughat," 2017) and Farhang-e-Talaffuz (2017) whereas English pronunciation was checked from Oxford Advanced Learners 'Dictionary 10th ed. (2020) and a comparison was made to highlight phonemic variation. Analysis of data indicates that almost one third loanwords retain their pronunciation and among the rest, the majority of words undergo change of vowel sounds whereas others either change a consonant sound or vowel and consonant both in some cases.

Key words: loanwords, phonemic variation, standard British English, Urdu

\section{INTRODUCTION}

\subsection{Background to the study}

Language contact is a very common phenomena and in a contact situation, one language influences another that results in linguistic change at different levels such as phonology, morphology, syntax and semantics etc. One such change is linguistic borrowing that is one of 
the most common sources of new words in languages. It refers to the process whereby speakers introduce words from one language into another (Trudgill, 1992) and these borrowings are usually known as loanwords (Crystal, 2008).

According to Dictionary.com (2020) English is one of the most incredible, flavourfully complex melting pots of loanwords from other countries that's been left to simmer for centuries. It has been borrowing words from other languages since its infancy. French, German, Greek, Spanish, African languages and Japanese are prominent donor languages to English as cited by Bielenia-Grajewska (2009). Other languages also contribute loanwords into English. In this regard Dictionary.com (2020) states, "As many as 350 other languages are represented and their linguistic contributions actually make up about 80\% of English". The present study is about Urdu loanwords and the phonemic variation they undergo into English Language.

\subsection{Statement of the Problem}

The English language has borrowed a lot of words from other languages. These loanwords undergo certain changes. The present study is about nature and phonemic variation of Urdu loanwords into standard British English.

\subsection{Aims and Objectives}

The objectives of the research are:

1) To find Urdu loanwords in standard British English

2) To identify phonemic variation of Urdu words in standard British English

\subsection{Research Questions}

The research answers following questions based on the objectives of the study:

1) Which Urdu words have been borrowed in standard British English?

2) Which phonemic changes do Urdu loanwords undergo in English language?

\subsection{Significance of the Study}

The present study is significant, because it focuses on phonemic variation of Urdu loanwords into standard British English. Earlier studies have explored loanwords and borrowing from English language to other languages or language varieties. Few studies have explored borrowings or loanwords into the English language and it makes the present study unique and different from previous studies. Moreover, it may urge linguists to explore loanwords from other languages into standard British English. 


\subsection{Limitation of the Study}

The research is limited to explore Urdu loanwords into standard British English and is delimited to a list of $135 \mathrm{f}$ words of daily use to see phonemic changes they come across in English. Changes at other linguistic levels i-e semantics, pragmatics are not the focus of the study.

\section{LITERATURE REVIEW}

Urdu is an Indo-Aryan language and is spoken mainly in South Asia. It is native to Pakistan and India. It serves as national language of Pakistan and is spoken by $7.08 \%$ people as a first language but is understood by a vast majority ("Population Census," 2017). According to Ethnologue, Urdu is spoken as a first and second language by 170 million people that makes it the $11^{\text {th }}$ most widely spoken language in the world (Simons \& Fennig, 2018). These countries include, Afghanistan, Australia, Behrain, Bangladesh, Canada, Denmark, Fiji, Finland, Germany, Guyana, Iran, Iraq, Malaysia, Mauritius, Nepal, New Zealand, Norway, Oman, Qatar, Saudi Arabia, Singapore, South Africa, Sweden, Turkey, United Kingdom and United States (Simons \& Fennig, 2018).

English belongs to the West Germanic branch of the Indo European family and serves as an international language. It is spoken in many countries both as a native and second or foreign language (Sipra, 2013). It is also spoken habitually in many countries of the world which include the United States, Canada, Australia, New Zealand, Ireland, the British Isles, Liberia, and many territories under the United Kingdom and the United States of America (Simons \& Fennig, 2018).

Urdu and English have been in contact with each other for more than four centuries (Rafi, 2013) and have borrowed many words from each other. Although loanwords from English to Urdu is a very common practice at present yet many words have entered into English language and have paved their way to be part of the standard English dialect. One language borrows words from another language in different ways. Sipra (2013) discusses three ways cited by Hasan and Khan (2019) as below:
i. Direct borrowing with a minor or no change in the word
ii. Translation of already existing word in the local language
iii. Mixing of vernacular and the foreign language

The present study analyses loanwords of the Urdu language that fall into the first category. In this case, the recipient language either tries to adopt loanwords with the help of sounds closest to the original sounds that words possess in the source language or sometimes with some 
phonological changes in the words (Sipra, 2013). This phenomenon is termed as adoption and adaptation (Al-Qinal, 2002). The original form and pronunciation of loanwords are maintained according to the donor language in adoption whereas loanwords are changed phonologically in adaptation. Substitution, deletion, addition, assimilation, and epenthesis are some common phonological adaptation processes (Hasan \& Khan, 2019).

English and Urdu both have phonological differences in their systems. English possesses fortyfour basic phonemes grouped into twenty-four consonants and twenty vowels. Roach (2013) classifies vowels into seven short vowels, five long vowels and eight diphthongs. In addition to the basic phonemes, Roach (2013) adds five triphthongs and two medial sounds $/ \mathbf{i} /, / \mathbf{u} /$ to the English phonemic inventory. On the contrary, the Urdu language has a rich inventory of phonemes. Bukhari $(1985,1991)$ lists twenty-four consonants, seven long vowels and seven short vowels. Khan (1997) describes ten basic vowels including three short/I, $\boldsymbol{\partial}, \boldsymbol{\mho} /$ and seven long $/ \mathbf{i}, \mathbf{e}, \mathbf{a}, \mathbf{a}, \mathbf{0}, \mathbf{o}, \mathbf{u}$ / vowels and forty-one consonants. Both of them discuss ten (five short $\&$ five long) nasalised vowels too in addition to basic vowels and consonants. Narang (2007) describes thirty-seven consonants and ten basic vowels. All these authors agree upon twentyeight consonant sounds whereas $/ \mathbf{P}, \mathbf{t}^{\mathbf{h}}, \mathbf{r h}, \mathbf{n}^{\mathbf{h}}, \mathbf{m}^{\mathbf{h}}, \mathbf{l}^{\mathbf{h}}, \mathbf{y} /$ are controversial sounds (Saleem et al., 2002). These studies clearly indicate that phonology of the Urdu language is different from that of English. Firstly, the number of phonemes are different in both languages. Secondly, all Urdu vowels are usually nasalised when they are used with nasal consonants $/ \mathbf{m}, \mathbf{n}, \mathbf{n}, \mathbf{n} /$ whereas this nasalisation feature is absent in the English language. Thirdly, Urdu does not possess documented diphthongs and triphthongs like English. Lastly, syllabification patterns of both languages also differ. Urdu does not follow the syllabic model of the English language. It allows zero or one consonant at onset position however a maximum of two consonants cluster at the coda position in particular cases (Usman, Ali, \& Masood, 2003). Urdu is considered to be a syllable-timed language and it allows vowel sounds to break words into syllables (N. W. Khan, Rizvi, \& Farooq, 2020). English speakers are supposed to omit vowel sounds to form complex clusters at the coda or termination position while pronouncing Urdu loanwords.

Loanword adaptations have been a matter of interest for many researchers for a long time. Hasan and Khan (2019) have cited a few recent studies in this regard which include loanword adaptation in Mandarin Chinese conducted by Miao (2005), Kenstowicz and Suchato (2006)'s study on issues in loanword adaptation, English loanwords in the spoken Arabic of the southern part of Iraq carried out by Abdullah and Daffar (2006), influence of orthography on loanword adaptations (Vendelin \& Peperkamp, 2006), English loanwords in Burmese explored by Chang (2009), phonotactic adaptation of English loanwords in Arabic (Al-Athwary, 2017) and morphological adaptation of English loanwords in twitter conducted by Dashti and Dashti (2017) etc. In the Pakistani context, studies like Morphophonemics of loanwords in translation (Al-Qinal, 2002), vowel substitution: a comparative study of English loans in Punjabi and Urdu (Hussain, Mahmood, \& Mahmood, 2011), phonological adaptation of English loanwords 
International Journal of Innovation, Creativity and Change. www.ijicc.net

Volume 15, Issue 8, 2021

in Pahari (A. Q. Khan \& Bukhari, 2011), phonological adaptations of English words borrowed into Punjabi (Mahmood, Hussain, \& Mahmood, 2011), phonological make-up of English loanwords incorporated into Punjabi via Urdu (Hussain, Mahmood, \& Mahmood, 2012), Urdu loanwords in Pakistani English (Bilal, Warraich, Fatima, Tiwana, \& Bhatti, 2012), linguistic study of borrowings from English to Urdu (Sipra, 2013), morphology of loanwords in Urdu (Mangrio, 2016), phonological treatment of vowels in English loanwords by Saraiki speakers(Atta, Syed, \& Bughio, 2017), phonological analysis of English loanwords in Dhani (Hasan \& Khan, 2019) and the study of English loanword phonology in Urdu (N. W. Khan et al., 2020) are some of the recent studies on Pakistani languages. Previously English loanwords into different languages have been explored by many researchers but there are few researches on loanwords into English language. The present research aims to fill this gap. Above mentioned studies are significant because they urged researchers to study phonemic variation of Urdu loanwords that standard British English borrows.

\section{METHODOLOGY}

\subsection{Nature of Research}

The present study follows a mixed method approach descriptive research design to identify phonemic variation of Urdu loanwords borrowed into standard British English.

\subsection{Population and Sample}

All Urdu words borrowed into English were the population of the study. A sample of 135 words of daily use was selected purposively from the Oxford Advanced Learners' Dictionary, 10th ed. (2020) for analysis.

\subsection{Framework}

Levenshtein's (1966) distance algorithm was followed to identify comparisons between Urdu and English speakers' pronunciation of Urdu loanwords. The framework has already been followed by Sanders and Chin (2009), Garcia and Yapici (2014), Chohan and García (2019) and Hasan and Khan (2019). Figure 2 below illustrates the algorithm:

$$
\operatorname{lev}_{a, b}(i, j)= \begin{cases}\max (i, j) & \text { if } \min (i, j)=0, \\
\min \left\{\begin{array}{l}
\operatorname{lev}_{a, b}(i-1, j)+1 \\
\operatorname{le}_{a, b}(i, j-1)+1 \\
\operatorname{lev}_{a, b}(i-1, j-1)+1_{\left(a_{i} \neq b_{j}\right)}
\end{array}\right.\end{cases}
$$

\section{Figure 2. The Levenshtein Algorithm adopted from Hasan and Khan (2019)}

This algorithm indicates the difference between two sequences. According to it, distance 
between two words is the minimum number of single-character edits (insertions, deletions or substitutions) required to change one word into the other. Distance value in present study indicates the number of phonemic variations that a word undergoes.

\subsection{Coding and Transcription}

All words were transcribed into IPA and their etymology was confirmed from the dictionary. Pronunciation and etymology of Urdu words were checked from "Urdu Lughat," (2017) and Farhang-e-Talaffuz (2017) whereas the Oxford Advanced Learners' Dictionary (2020) was used to confirm pronunciation and English transcription of Urdu loanwords and a comparison was made to highlight phonemic variation. The data was arranged in a table (4.1) consisting serial number, Urdu transcription, English transcription, distance value and gloss as shown below:

Table 4.1 Urdu loanwords in standard British English

\begin{tabular}{|c|c|c|c|c|c|}
\hline $\mathrm{Sr}$ & Urdu Transcription & English Transcription & Distance Value & Gloss & \\
\hline 1 & /mu:li/ & /mu:li/ & 0 & Mooli & مولى \\
\hline 2 & /na: n/ & /na:n/ & 0 & Nan & 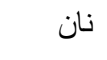 \\
\hline 3 & /pa:n/ & /pa:n/ & 0 & Paan & 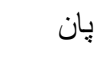 \\
\hline 4 & /a:pə/ & /a:pə/ & 0 & Aapa & آبا \\
\hline 5 & /t $\int a \mathrm{a} /$ & /t $\int a \mathrm{a} /$ & 0 & Chai & جائح \\
\hline 6 & /aia/ & /aia/ & 0 & Ayah & آير \\
\hline 7 & /ba:ba:/ & /ba:ba:/ & 0 & Baba & بابا \\
\hline 8 & /ba:bu:/ & /ba:bu:/ & 0 & Babu & بابو \\
\hline 9 & 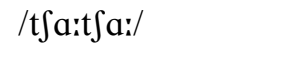 & 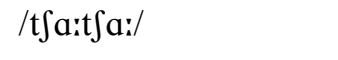 & 0 & Chacha & جاجا \\
\hline 10 & /pu:ri/ & /pu:ri/ & 0 & Puri & يو ليورى \\
\hline 11 & /na:/ & /na:/ & 0 & $\mathrm{Nah}$ & 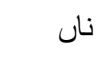 \\
\hline 12 & /səla:m/ & /səla:m/ & 0 & Salaam & 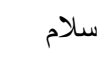 \\
\hline 13 & / $a: b a: \int /$ & 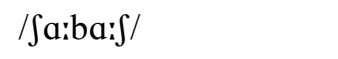 & 0 & Shabash & 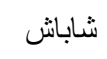 \\
\hline 14 & /su:fi/ & /su:fi/ & 0 & Sufi & صوفى صى \\
\hline 15 & /nəwa:b/ & /nəwa:b/ & 0 & Nawab & 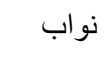 \\
\hline 16 & /dzwa:n/ & /dzwa:n/ & 0 & Jawan & جوان \\
\hline 17 & /musaiərə/ & /mufaiərə/ & 0 & Mushaira & مشاعره \\
\hline 18 & /kisa:n/ & /kisa:n/ & 0 & Kisan & كسان \\
\hline 19 & /lu:t/ & /lu:t/ & 0 & Loot & لوث \\
\hline 20 & / Sa:mia:nə/ & / sa:mia:nə/ & 0 & Shamiana & شاميان: \\
\hline 21 & /nai/ & /nai/ & 0 & Nai & نائً \\
\hline 22 & /a:lu:/ & /alu:/ & 1 & Aloo & آلو \\
\hline 23 & / da:l/ & /da:l/ & 1 & $\mathrm{Dal}$ & دال \\
\hline
\end{tabular}


International Journal of Innovation, Creativity and Change. www.ijicc.net

Volume 15, Issue 8, 2021

\begin{tabular}{|c|c|c|c|c|c|}
\hline $\mathrm{Sr}$ & Urdu Transcription & English Transcription & Distance Value & Gloss & \\
\hline 24 & /gərəm məsa:lə/ & /gurəm məsa:lə/ & 1 & $\begin{array}{l}\text { Garam } \\
\text { masala }\end{array}$ & كرم مصالح \\
\hline 25 & /huqə/ & /hukə/ & 1 & Hookah & حق \\
\hline 26 & /lasi/ & /læsi/ & 1 & Lassi & 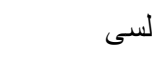 \\
\hline 27 & /pəkə:tə/ & /pəkə:ra/ & 1 & Pakora & يكوزْه \\
\hline 28 & /pəra:t $\mathbf{t}^{\mathrm{h}} \mathrm{g} /$ & /pəra:tə/ & 1 & Paratha & بر إثها \\
\hline 29 & /raițo/ & /raitə/ & 1 & Raita & رائت \\
\hline 30 & $/$ ro:ti/ & /routi/ & 1 & Roti & 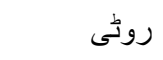 \\
\hline 31 & $/ s \mathbf{a} g /$ & $/ \mathrm{sæg} /$ & 1 & Saag & 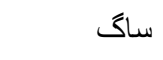 \\
\hline 32 & /səbzi:/ & /sıbzi:/ & 1 & Sabzi & سبزى \\
\hline 33 & /səmə:sə/ & /səməusə/ & 1 & Samosa & سموسـ \\
\hline 34 & / ț țikə/ & /ttıkə/ & 1 & Tikka & تكى \\
\hline 35 & /qəmi:z/ & /kəmi:z/ & 1 & Kameez & قميض \\
\hline 36 & /pa:dza:mə/ & /pədza:mə/ & 1 & Pajama & لِاجام \\
\hline 37 & /dzolesbi/ & /dzəlerbi/ & 1 & Jalebi & جلييى \\
\hline 38 & $/ \int \mathbf{a}: 1 /$ & $/ \int \mathfrak{9}: 1 /$ & 1 & Shawl & 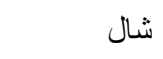 \\
\hline 39 & /to:pi/ & /tәopi/ & 1 & Topi & لثويى \\
\hline 40 & /aba/ & /aba/ & 1 & Abba & ابا \\
\hline 41 & /əma:/ & /ıma:/ & 1 & Amma & امان \\
\hline 42 & $/ d_{3} \mathfrak{3}: \mathbf{s} /$ & $/ \mathrm{d}_{3} \mathbf{p} \mathbf{s} /$ & 1 & Josh & جوش \\
\hline 43 & /lungi// & /lungi:/ & 1 & Lungi & لنكى \\
\hline 44 & /bergəm/ & /bergəm/ & 1 & Begum & بيكم \\
\hline 45 & $/ \mathbf{b}^{\mathrm{h}} \mathrm{aI} /$ & /bai/ & 1 & Bhai & بهائً \\
\hline 46 & /ja:r/ & /ja:/ & 1 & Yaar & يار \\
\hline 47 & /ba:y/ & /ba:g/ & 1 & Bagh & باغ \\
\hline 48 & /ba:za:r/ & /bəza:r/ & 1 & Bazaar & باز ار \\
\hline 49 & $/ \mathbf{d}^{\mathrm{h}} \mathrm{a}: \mathrm{bə} /$ & /da:ba/ & 1 & Dhaba & دذهابر \\
\hline 50 & /d] 3 Imxa:nə/ & /dżImka:nə/ & 1 & Gymkhana & جم خان \\
\hline 51 & /mo:rtfa/ & /mo:t5a/ & 1 & Morcha & مورجه \\
\hline 52 & /nailan/ & /nailpn/ & 1 & Nylon & نائَلْن \\
\hline 53 & /pəך|k $\mathbf{k}^{\mathbf{h}}$ / & $/ \mathrm{p} \Lambda \mathrm{\eta} \mathbf{k} \boldsymbol{2} /$ & 1 & Punkah & ينكها \\
\hline 54 & / tha:li/ $^{\mathrm{h}} \mathrm{d}$ & /ta:li/ & 1 & Thali & تهالى \\
\hline 55 & /me:la/ & /meila/ & 1 & Mela & ميلا \\
\hline 56 & /xa:n/ & /ka:n/ & 1 & Khan & خان \\
\hline 57 & /pæsa:/ & /paisa:/ & 1 & Paisa & بيس \\
\hline
\end{tabular}


International Journal of Innovation, Creativity and Change. www.ijicc.net

Volume 15, Issue 8, 2021

\begin{tabular}{|c|c|c|c|c|c|}
\hline $\mathrm{Sr}$ & Urdu Transcription & English Transcription & Distance Value & Gloss & \\
\hline 58 & $/ \mathrm{rIk} \int \mathbf{a} /$ & $/ \mathrm{rIk} \int \mathbf{s} \mathbf{z} /$ & 1 & Rickshaw & ركثش \\
\hline 59 & /xa:ki/ & /ka:ki/ & 1 & Khaki & خاكى \\
\hline 60 & /bədma:S/ & /bsdma: $\int /$ & 1 & Badmash & بدمعاش \\
\hline 61 & /pətwa:ri/ & /pstwa:ri/ & 1 & Patwari & 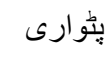 \\
\hline 62 & $/ \mathbf{g}^{\mathbf{h}_{\mathrm{i}} / /}$ & $/ \mathbf{g i} / /$ & 1 & Ghee & كهى \\
\hline 63 & /qulfi/ & /kulfi/ & 1 & Kulfi & قلفى \\
\hline 64 & /dzəygli/ & /dżngli/ & 1 & Jungli & جنكلى \\
\hline 65 & /kəbədi/ & /ksbədi/ & 1 & Kabaddi & كبذى \\
\hline 66 & $/ \mathbf{k}^{\mathbf{h}} \mathrm{d}: \mathrm{di} /$ & /ka:di/ & 1 & Khadi & 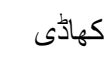 \\
\hline 67 & /nəmək/ & /nımək/ & 1 & Namak & نمى \\
\hline 68 & $/ \mathbf{k}^{\mathbf{h}} \mathrm{a}: n ə /$ & /ka:nə/ & 1 & Khana & كهانا \\
\hline 69 & /kro:c/ & $/ \mathrm{kro}: \mathrm{r} /$ & 1 & Crore & كروز كر \\
\hline 70 & /məhəl/ & /məha:l/ & 1 & Mahal & 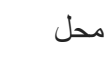 \\
\hline 71 & /nasla/ & /nılə/ & 1 & Nullah & ناله \\
\hline 72 & /țəma:Sə/ & /təma:Sə/ & 1 & Tamasha & تماشد \\
\hline 73 & /bətfə/ & $/ \mathrm{b} \boldsymbol{\Lambda} \mathrm{t} \int \mathrm{a} /$ & 1 & Bachcha & بج: \\
\hline 74 & $/ \mathbf{x} \cup \int \mathrm{I} /$ & $/ \mathbf{k} v \int \mathrm{I} /$ & 1 & Cushy & 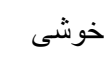 \\
\hline 75 & /dzuga:t/ & /dzuga:d/ & 1 & Jugaad & جكاز \\
\hline 76 & /kița:b/ & /kita:b/ & 1 & Kitab & كتاب \\
\hline 77 & /suni/ & /suni/, /ssni/ & 1 & Sunni & سنى \\
\hline 78 & /mula/ & /mulə/, /mslə/ & 1 & Mullah & ملا \\
\hline 79 & /bersən/ & /beisın/ & 2 & Besan & بيسن \\
\hline 80 & /bərja:ni/ & /bıria:ni/ & 2 & Biryani & 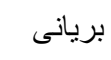 \\
\hline 81 & 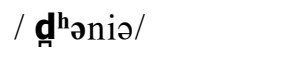 & /da:niə/ & 2 & Dhania & دهنيا \\
\hline 82 & /qo:rmə/ & /ko:mə/ & 2 & Korma & قورم ق ق ق \\
\hline 83 & /əna:/ & /Ana/ & 2 & Anna & انا انا \\
\hline 84 & /ət Jkən/ & /ASkən/ & 2 & Achkan & اجّكن \\
\hline 85 & /burqa/ & /buəkə/, /bз:kə/ & 2 & Burqa & برقع \\
\hline 86 & /dupuptə/ & /dupstə/ & 2 & Dupatta & دويلث \\
\hline 87 & /nəqa:b/ & /nıka:b/ & 2 & Niqab & نقاب \\
\hline 88 & /Se:rwa:ni/ & 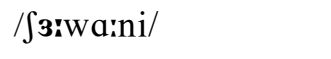 & 2 & Sherwani & شيروانى \\
\hline 89 & /bənd $\mathbf{d}^{\mathrm{h}} /$ & $/ \mathrm{b} \boldsymbol{\Lambda} \mathrm{nd} /$ & 2 & Bandh & بنده \\
\hline 90 & $/ \partial \int^{h_{a}} /$ & /st5a:/ & 2 & Achcha & اجها \\
\hline 91 & /alha:dza/ & /alhæadzə/ & 2 & Alhaja & الحاجـ \\
\hline 92 & /alha:dzi/ & /alhæedzi// & 2 & Alhaji & الحاجى \\
\hline
\end{tabular}


International Journal of Innovation, Creativity and Change. www.ijicc.net

Volume 15, Issue 8, 2021

\begin{tabular}{|c|c|c|c|c|c|}
\hline $\mathrm{Sr}$ & Urdu Transcription & English Transcription & Distance Value & Gloss & \\
\hline 93 & /mufți/ & $/ \mathrm{m} \Delta \mathrm{fti} /$ & 2 & Mufti & مفتى \\
\hline 94 & /mu:dza:hədiin/ & /mu:dzəhədi:n/ & 2 & Mujahideen & مجاحدين \\
\hline 95 & /t $\int ә p t a: s i: /$ & $/ \mathrm{t} \int \Lambda$ pra:si:/ & 2 & Chaprasi & 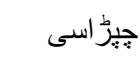 \\
\hline 96 & /tfa:rpai/ & 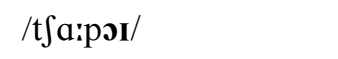 & 2 & Charpoy & جاريائً \\
\hline 97 & /sa:hib/ & /sa:b/, /sa:ıb/ & 2 & Sahib & صاحب \\
\hline 98 & /mehndidi/ & $/$ mendi/ & 2 & Mehndi & 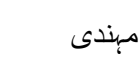 \\
\hline 99 & $/ \operatorname{la}: \mathbf{k}^{\mathbf{h}} /$ & $/ 1 \mathfrak{k} /$ & 2 & Lakh & لاكه \\
\hline 100 & / țəbla/ & /tablə/, /tıblə/ & 2 & Tabla & طبل \\
\hline 101 & /mũ:ng/ & /mu:y/ & 2 & Moong & مونى \\
\hline 102 & /bəhu/ & /ba:hu:/ & 2 & Bahu & بهو \\
\hline 103 & $/ \operatorname{mirts} /$ & $/ \mathrm{m} 3 \mathrm{t} \mathrm{t} /$ & 2 & Mirch & مرج \\
\hline 104 & /wlarți/ & /blaiti/ & 2 & Blighty & 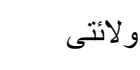 \\
\hline 105 & /pəy $\mathbf{k}^{\mathbf{h}}$ ə/ & /рмұkә/ & 2 & Punkah & بنكها \\
\hline 106 & $/ \mathbf{t}^{\text {h }}$ əg/ & $/ \theta \boldsymbol{A} \mathrm{g} /$ & 2 & Thug & ثهى \\
\hline 107 & /quli/ & /ku:li/ & 2 & Coolie & قلى \\
\hline 108 & $/ \mathbf{t} \int^{\mathrm{h}} \mathbf{O}: \mathbf{t a} /$ & /țəutə/ & 2 & Chota & جهونا \\
\hline 109 & /mæda:n/ & /maida:n/ & 2 & Maidan & 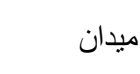 \\
\hline 110 & /usța:d / & /usta:d/ & 2 & Ustad & 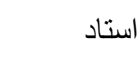 \\
\hline 111 & /ba:da:m/ & /bıda:m/, /ba:da:m/ & 2 & Badam & بادام \\
\hline 112 & /pənda:l/ & /pændl/, /pænda:l/ & 2 & Pandal & يندال \\
\hline 113 & /dersi/ & /dersi/ & 2 & Desi & 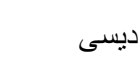 \\
\hline 114 & /go: $\int \mathbf{t} /$ & /gəuSt/ & 2 & Gosht & كوشت \\
\hline 115 & 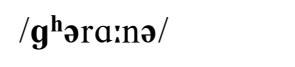 & /gıra:nə/ & 2 & Gharana & كهر انـ \\
\hline 116 & $/ \mathbf{d} \mathbf{z}^{\mathbf{h}} \mathbf{u g i} /$ & /dzıgi/ & 2 & Jhuggi & جهىى \\
\hline 117 & /da:da:/ & /da:da:/ & 2 & Dada & دادا \\
\hline 118 & /xəri:f/ & /ks ri:f/ & 2 & Kharif & خريف \\
\hline 119 & /tfa:dar/ & /tfa:do: $\mathbf{r}^{\mathrm{r} /}$ & 3 & Chador & جادر \\
\hline 120 & 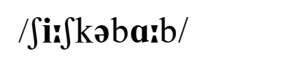 & /SIIfkrbæab/ & 3 & Shish kebab & شيش كباب \\
\hline 121 & / d $\mathbf{d}^{\mathbf{h}} \mathbf{o}: \mathrm{t} \mathrm{i}$ / & /dəuti/ & 3 & Dhoti & 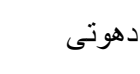 \\
\hline 122 & /kurțo/ & /k3:tə/ & 3 & Kurta & 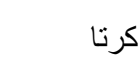 \\
\hline 123 & /pərdda/ & /pз:da/ & 3 & Purdah & يرده \\
\hline 124 & /Jəlwa:r/ & /ss lwa: ${ }^{\mathrm{r}} /$ & 3 & Salwar & 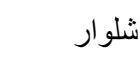 \\
\hline 125 & /məsihə/ & /məsara/ & 3 & Messiah & مسيحا \\
\hline 126 & /țolwa:r/ & $/ \mathbf{t} \mathbf{\Lambda} \mathbf{l w a}^{\mathrm{r} /}$ & 3 & Talwar & تلكوار \\
\hline 127 & /sərka:r/ & /sз:ka:r/ & 3 & Sarkar & سركار \\
\hline
\end{tabular}


International Journal of Innovation, Creativity and Change. www.ijicc.net

Volume 15, Issue 8, 2021

\begin{tabular}{|c|c|c|c|c|c|}
\hline $\mathrm{Sr}$ & Urdu Transcription & English Transcription & Distance Value & Gloss & \\
\hline 128 & /sulța:n/ & /saltan/ & 3 & Sultan & سلطان \\
\hline 129 & 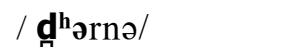 & /d3:nə/ & 3 & Dharna & 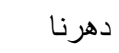 \\
\hline 130 & /bəygləa/ & /bsygələu/ & 3 & Banglow & بنكل \\
\hline 131 & /mu:hələ/ & /məohsl3:/ & 3 & Mohalla & محل \\
\hline 132 & /xa:nsa:mã̃: / & /ka:nsəma:/ & 3 & Khansama & خانسامان \\
\hline 133 & /məzdu:r/ & $/ \mathrm{m} \boldsymbol{\Delta} z \mathbf{d} \boldsymbol{u} \boldsymbol{\partial}^{\mathrm{r}} /$ & 4 & Mazdoor & مزدور \\
\hline 134 & /kəmərbənd / & $/ \mathrm{k} \boldsymbol{\Delta} \mathrm{m} ə \mathrm{~b} \boldsymbol{\Lambda} \mathrm{nd} /$ & 4 & Cummerbund & كمر بند \\
\hline 135 & / țəndururi/ & /tænduəri/, /tænduri/ & 4 & Tandoori & 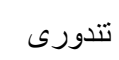 \\
\hline
\end{tabular}

\section{DATA ANALYSIS}

Data presented in table 4.1 above clearly shows that standard British English borrowed loanwords from the Urdu language. These words have been borrowed from different fields like edibles, clothing, relations, places, professions, conversation and miscellaneous. Column 4 in table 4.1 indicates distance values in terms of phonemic variation of Urdu loanwords in standard British English. Zero distance value means no variation in phonemes. Distance values $1,2,3$ and 4 identify the number of phonemes that undergo change in the process of borrowing. Analysis of the selected corpus shows that Urdu loanwords can be broadly classified into two groups i-e words that do not change their pronunciation and words which change their pronunciation. Figure 3 below, sums up the Levenshtein Distance analysis of Urdu loanwords in standard British English:

\section{Percentage of words

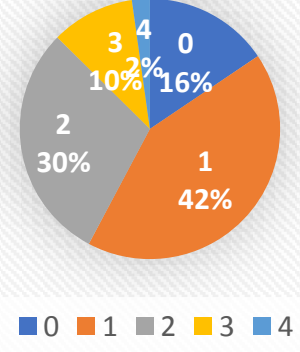

Figure 3. Levenshtein Distance Analysis

Detailed analysis of the data shows discrete changes in phonological patterns of Urdu loanwords and elaborates distance value in detail. Description of data is based on categories ie substitution, deletion, addition and epenthesis in accordance with distance value. The detailed description is as below: 


\subsection{No Change}

Twenty-one words (16\%) at serial 1-21 in table 4.1 indicate zero (0) Levenshtein distance value. This means these words do not undergo any phonemic change during borrowing and they retain their pronunciation.

\subsection{One Change}

Fifty-seven words i-e 42\% shown at serial 22-78 in table 4.1 show one (1) Levenshtein distance value. This indicates that these words undergo one phonemic change. Comparison of Urdu and English words at serial 22-78 in table 4.1 shows that this phonemic change is either a substitution of a vowel or consonant or deletion of a consonant sound. Their detail is as below:

\subsubsection{Substitution of Vowel}

Data shows that substitution of Urdu vowels is the dominant adaptation strategy in English language. Analysis of data shows that standard British English substitutes some vowel sounds of Urdu loanwords. Examples of vowel substitution from table 4.1 are as below:

i. Urdu short vowel / $\mathbf{a} /$ is replaced with English central vowel / $\mathbf{N} /$ as in words garam masala, sabzi, abba, amma, badmash, patwari, jungle, kabaddi, namak, bachcha (sr. $24,32,40,41,60,61,66,65,67,73$ ) and sometimes it is substituted with /ax/ as sown in word lassi (sr.26). It can be found substituted with short vowel /p/ as in word nylon (sr.52). Sometimes the same short vowel is replaced with English back long vowel /o:/ and back long vowel /a:/ as shown in words rickshaw, mahal respectively (sr. 58, 70).

ii. Urdu long vowel /a:/ is replaced with English central vowel /ə/ as in words pajama, bazar, nullah (sr. 36, 48, 71). In some words, the same vowel is substituted with English front short vowel /a/ as in words aloo, saag (sr. 22, 31). Sometimes the same vowel is found substituted with English back long vowel /o:/ as in word shawl (sr. 38).

iii. Urdu long vowel /o:/ is substituted with English closing diphthong/əひ/ as in words roti, samosa and topi (sr. 30, 33, 39). The same vowel is also substituted with English back short vowel /p/ as in word josh (sr. 42).

iv. Urdu short vowel /i/ is replaced with English front close long vowel/ii/ as in word lungi (sr. 43).

v. Urdu long vowel /e:/ is changed with English closing diphthong /eI/ as in words jalebi, begum and mela (sr. 37, 44, 55).

vi. Urdu short vowel /a/ is replaced with English closing diphthong /aI/ as in example word paisa (sr. 57).

vii. Urdu back short vowel / $\mathbf{U} /$ is substituted with English central vowel / $\mathbf{N}$ as shown in words sunni and mullah (sr. 77, 78). 


\subsubsection{Substitution of Consonant}

The English language does not have as rich repository of consonant sounds as Urdu has. English speakers adapt those consonants of Urdu language to the nearest possible consonant sounds of the English language. Cases of substitution of consonant sounds of Urdu loanwords in standard British English from collected data (table 4.1) are as below:

i. Urdu voiced dental stop /d/ is substituted with English voiced alveolar stop /d/ as in word dal (sr. 23).

ii. Urdu voiceless, aspirated alveolar stop $/ \mathbf{t}^{\mathbf{h}} /$ is replaced with English voiceless alveolar stop /t/ as in word pratha (sr. 28).

iii. Urdu voiced, aspirated alveolar stop $/ \mathbf{d}^{\mathbf{h}} /$ is replaced with English voiced alveolar stop /d/ as in word dhaba (sr. 49).

iv. Urdu unaspirated voiceless dental stop /ț/ is replaced with English voiceless alveolar stop /t/ as in words raita, tikka, tamasha and kitab (sr. 29, 34, 72, 76). Similarly, Urdu voiceless aspirated stop $/ \mathbf{t}^{\mathbf{h}} /$ is replaced with English voiceless alveolar stop $/ \mathbf{t} /$ as in word thali (sr. 54).

v. Urdu voiced, aspirated bilabial stop $/ \mathbf{b}^{\mathbf{h}} /$ is substituted with English voiced bilabial stop /b/ as in word bhai (sr. 45).

vi. Urdu uvular voiceless stop /q/ is replaced with English voiceless velar stop / $\mathbf{k} /$ as in words hookah, kameez and kulfi (sr. 25, 35, 63).

vii. Urdu voiceless velar fricative $/ \chi /$ is substituted with English voiceless velar stop $/ \mathbf{k} /$ as in words gymkhana, khan, khaki and cushy (sr. 50, 56, 59, 74).

viii. Urdu voiceless, aspirated velar stop $/ \mathbf{k} \mathbf{h} /$ is substituted with English voiceless velar stop /k/ as in words punkah, khadi and khana (sr. 53, 66, 68).

ix. Urdu voiced velar fricative $/ \mathbf{\gamma} /$ is substituted with English voiced velar stop $/ \mathbf{g} /$ as in word bagh (sr. 47).

x. Urdu voiced, aspirated velar stop $/ \mathbf{g}^{\mathbf{h}} /$ is substituted with English voiced velar stop /]/ as in word ghee (sr. 62).

xi. Urdu voiced retroflex flap $/ \mathbf{r} /$ is substituted with English palato-alveolar approximant $/ \mathbf{r} /$ as in word pakora (sr.27). The same consonant can be found replaced with English voiced alveolar /d/ as in word jugaad (sr. 75).

\subsubsection{Deletion}

Deletion or elision is omission of one or more sounds in a word. In the English language, post alveolar approximant $/ \mathbf{r} /$ at syllable coda position is omitted when it is followed by a short vowel /ə/. Urdu voiced retroflex trill /r/ used in Urdu loanwords is deleted in standard British English as in words yaar, morcha (sr. 46, 51). Another case of such sort is deletion of Urdu 
voiced retroflex flap / $\mathbf{t}$ as in word crore (sr. 69).

\subsection{Two Changes}

$30 \%$ words shown at serial 79-118 in table 4.1 from data show Levenshtein distance value 2 (two). It means, two phonemics changes occur in these words. The most prominent of these changes is double substitution. In this pattern two sounds such two vowels, two consonants or a vowel and a consonant are substituted in a word. Description of such cases with examples from data is as below:

\subsubsection{Substitution of two vowels:}

In this pattern, two Urdu vowels are replaced such as:

i. If an Urdu long vowel /e:/ and /ə/ are used in a word together, /e:/ is replaced with an English closing diphthong /eI/ and /a/ is replaced with an English central short vowel $/ \mathbf{\Lambda} /$ as in word besan (sr. 79).

ii. If an Urdu central short vowel /ə/ is used in the first syllable and an Urdu long vowel /e:/ is used in the second syllable of a word like biryani (sr. 80), /a/ is replaced with an English front closing vowel /I/ and /e:/ is replaced with an English medial weak vowel $/ \mathbf{i} /$.

iii. When an Urdu central short vowel /a/ is used with an Urdu long vowel /a:/ together, $/ \boldsymbol{\partial} /$ is substituted with an English central short vowel $/ \mathbf{\Lambda} /$ whereas a long vowel /a: $/$ is substituted with an English central weak vowel /o/ as in word anna (sr. 83). Sometimes the same combination of Urdu vowels is replaced with English front open short vowels $/ \mathfrak{a} /$ as in words alhaji and alhaji (sr. 91, 92).

iv. If two Urdu short vowels /a/ and / $\mathbf{0} /$ come together in a word, the initial vowel /a/ is changed with an English open back long vowel /a:/ and the second vowel is changed with an English close back long vowel /us/ as in word bahu (sr. 102).

\subsubsection{Substitution of two consonants}

In this pattern, two Urdu consonants are substituted such as:

i. If an Urdu labiodental voiced fricative /w/ and an Urdu voiceless unaspirated dental stop / $/ \mathbf{n} /$ are used in a word together, /w/ is replaced with an English voiced bilabial stop /b/ and / t/ / is substituted with an English voiceless alveolar stop /t/ as in the word blighty (sr. 104).

ii. When an Urdu voiceless unaspirated dental stop /t t/ and an Urdu voiced unaspirated dental stop /d/ are used in a word, /t/t/ becomes an English voiceless alveolar plosive /t/ and /d/ becomes an English voiced alveolar plosive /d/ as in the word ustad (sr. 110). 
International Journal of Innovation, Creativity and Change. www.ijicc.net

Volume 15, Issue 8, 2021

iii. Sometimes an Urdu voiced unaspirated dental stop /d / is used twice in a word such as dada (sr. 117); it is substituted with an English voiced alveolar plosive /d/.

\subsubsection{Substitution of a vowel and a consonant}

In this pattern one vowel and one consonant sound are substituted in a word. They can be in one syllable or in two different syllables. Description of this pattern with examples from data is as below:

i. An Urdu central short vowel /o/ used in words achkan, dupatta, bandh, achcha, chaprasi, punkah, thug, gharana and kharif (sr. 84, 86, 89, 90, 95, 105, 106, 115, 118) is substituted with an English central short vowel / $\mathbf{N}$ and a consonant sound in each of these words is also substituted. An Urdu post alveolar unaspirated affricate /t $\mathbf{f} /$ in the word achkan (sr. 84) is changed with an English palate alveolar voiceless fricative / $\mathbf{S}$. An Urdu voiced, unaspirated dental stop /d/ in the word dupatta (sr. 86) is changed with an English voiced alveolar plosive /d/. An Urdu voiced, aspirated dental stop / $\mathbf{d}^{\mathbf{h}} /$ in the word bandh (sr. 89) is changed with an English voiced alveolar plosive /d/. An Urdu post alveolar aspirated affricate $/ \mathbf{t} \mathbf{~}^{\mathrm{h}} /$ in the word achcha (sr. 90) is changed with an English voiceless palate alveolar affricate $/ \mathbf{t} \mathbf{J} /$. An Urdu voiced retroflex flap $/ \mathbf{t} /$ in the word chaprasi (sr. 95) is substituted with an English palato-alveolar approximant $/ \mathbf{r} /$. An Urdu voiceless, aspirated velar stop $/ \mathbf{k}^{\mathbf{h}} /$ in the word punkah (sr. 105) is substituted with an English voiceless velar stop $/ \mathbf{k} /$. An Urdu voiceless, aspirated alveolar stop / $/ \mathbf{t}^{\mathbf{h}} /$ in the word thug (sr. 106) is substituted with an English voiceless dental fricative $/ \boldsymbol{\theta} /$. An Urdu voiced, aspirated velar stop $/ \mathbf{g}^{\mathbf{h}} /$ in the word gharana (sr. 115 ) is substituted with an English voiced dental stop /g/. An Urdu voiceless, velar fricative $/ \chi /$ in the word kharif (sr. 118) is substituted with an English voiceless velar stop /k/. In an Urdu word dhania (sr. 81) an Urdu central short vowel /ə/ is substituted with an English open back long vowel /a:/ and an Urdu voiced, aspirated dental stop /d $\mathbf{d}$ / is changed with an English voiced alveolar plosive /d/. In an Urdu word niqab (sr. 87) an Urdu central short vowel/ə/is substituted with an English front close short vowel $/ \mathbf{I} /$ and an Urdu uvular stop /q/ at the onset position of the second syllable is substituted with an English voiceless velar stop /k/. In the word tabla (sr. 100) an Urdu central short vowel /ə/ is substituted with an English front open short vowel /a/ and an Urdu unaspirated voiceless dental stop $/ \mathbf{t} /$ is replaced with an English voiceless alveolar stop $/ \mathbf{t} /$.

ii. An Urdu long vowel /a:/ in the second syllable of the word mujahideen (sr. 94) is substituted with an English central weak vowel /ə/ and an Urdu voiced, unaspirated dental stop /d / at the onset position of the fourth syllable is changed with an English voiced alveolar plosive /d/. In the word lakh (sr. 99) the same vowel is substituted with 
an English front open short vowel /å/ and an Urdu voiceless, aspirated velar stop / $\mathbf{k}$ / is substituted with an English voiceless velar stop /k/. In the word badam (sr. 111) the same vowel is replaced with an English central short vowel / $\mathbf{\Lambda}$ and an Urdu voiced, unaspirated dental stop /d $/$ / is changed with an English voiced alveolar plosive /d/.

iii. An Urdu back close short vowel / $/$ / in the words mufti and jhuggi (sr. 93, 116) is substituted with an English central short vowel / $/$. An Urdu unaspirated voiceless dental stop /ț/ in mufti (sr. 93) is replaced with an English voiceless alveolar stop /t/, whereas an Urdu aspirated, voiced post alveolar affricate $/ \mathbf{d} \mathbf{3}^{\mathbf{h}} /$ in $j h u g g i$ (sr. 116) is replaced with an English palate alveolar voiced affricate /d3/. The same vowel in the word coolie (sr. 107) is replaced with an English back close long vowel /u:/ whereas an Urdu uvular stop /q/ at the onset position of the first syllable is substituted with an English voiceless velar stop / $\mathbf{k} /$.

iv. An Urdu vowel /o:/ in the words chota and gosht (sr. 108, 114) is substituted with an English closing diphthong /ov/ and an Urdu post alveolar aspirated affricate $/ \mathbf{t} \int^{\mathrm{h}} /$ chota (sr. 108) is changed with an English voiceless palate alveolar affricate /t $\int /$ and an Urdu unaspirated voiceless dental stop /t/ in gosht (sr. 114) is replaced with an English voiceless alveolar stop /t/.

v. An Urdu front open short vowel /a/ in the word maidan (sr. 109) is substituted with an English closing diphthong /ai/ and an Urdu voiced, unaspirated dental stop /d $/$ is changed with an English voiced alveolar plosive /d/.

vi. An Urdu long vowel /es/ in the word desi (sr. 113) is substituted with an English closing diphthong /ei/ and an Urdu voiced, unaspirated dental stop /d / is changed with an English voiced alveolar plosive /d/.

Besides double substitution of phonemes in a word, sometimes one phoneme is substituted, and one is deleted as described below:

i. In some Urdu words one vowel sound is replaced and one sound is deleted, such as in the word pandal (sr. 112), a central short vowel /a/ in the first syllable is substituted with an English central short vowel / $\mathbf{N}$ and an Urdu long vowel /a:/ in the second syllable is deleted by some English speakers and some pronounce it without any change.

ii. Sometimes a vowel sound is substituted, and a consonant sound is deleted. In the Urdu word sherwani (sr. 88), an Urdu long vowel /e:/ in the first syllable is substituted with an English mid-central vowel /3:/ and an unaspirated alveolar trill $/ \mathbf{r} /$ is omitted at the termination of the first syllable. In the word charpoy (sr. 96), an Urdu unaspirated alveolar trill $/ \mathbf{r} /$ at the coda position of the first syllable is omitted and an Urdu diphthong /ai/ in the second syllable is replaced with an English closing diphthong /or/. 
In the word mirch (sr. 103), an Urdu short vowel /I/ is substituted with an English midcentral vowel /3:/ whereas an unaspirated alveolar trill /r/ in the coda cluster is omitted. In the word sahib (sr. 97) an Urdu glottal fricative /h/ at the onset position of the second syllable and an Urdu short vowel /I/ as the centre of the same syllable are omitted and the termination of this syllable becomes the coda of the first syllable. However, some English speakers pronounce it as /sa:ib/. In the word moong (sr. 101), an Urdu nasalised back long vowel /ũ:/ is replaced with an English back long vowel /u:/ and an Urdu voiced unaspirated velar stop $/ \mathbf{g} /$ in the coda cluster is deleted.

iii. Sometimes one consonant sound is substituted, and one consonant is deleted as in the word korma (sr. 82); an Urdu uvular stop /q/ at the onset position of the first syllable is substituted with an English voiceless velar stop /k/ and an unaspirated alveolar trill /r/ at the coda position of the same syllable is omitted. In the word mehndi (sr. 98), an Urdu glottal fricative /h/ is omitted and an Urdu voiced unaspirated dental stop /d $/$ is replaced with an English voiced alveolar plosive /d/.

\subsection{Three Changes}

$14 \%$ words shown at serial $119-132$ in table 4.1 show the Levenshtein distance value 3 (three). These words show three phonemic changes. Their detail with examples from data has been discussed as below:

i. Substitution of three vowels: Some words undergo substitution of three vowel sounds at the same time. In the word shish kebab (sr. 120) an Urdu close front long vowel /i:/ is replaced with an English close front short vowel /I/, an Urdu central vowel /a/ is substituted with an English close front short vowel /I/ whereas an Urdu back long vowel /a:/ is changed with an English open front short vowel/a/. Similarly, in the Urdu word mohalla (sr. 131) an Urdu close front long vowel /i:/ is substituted with an English

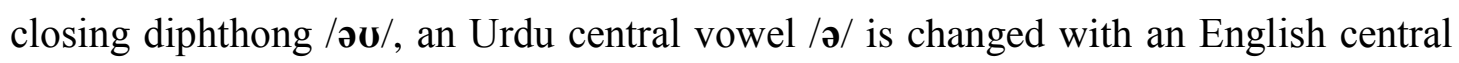
vowel / $\mathbf{A} /$ and the same Urdu vowel at the word ending position is replaced with an English mid-central vowel /3:/.

ii. Substitution of two consonants and a vowel: In this case two Urdu consonants and a vowel are substituted with English consonants and a vowel. For example, the Urdu word dhoti (sr. 121) shows this variation. An Urdu voiced aspirated dental stop /d/ is replaced with an English voiced alveolar plosive /d/, an Urdu back long vowel /o:/ is changed with an English closing diphthong /ov/ and an Urdu voiceless unaspirated dental stop /t $/$ / is replaced with an English voiceless alveolar stop /t/.

iii. Substitution of two vowels and a consonant: In this case two Urdu vowels and a consonant sound are replaced. The Urdu word sultan (sr. 128) is an example of such a 
variation where an Urdu back short vowel / $\mathbf{u}$ is replaced with an English central vowel $/ \mathbf{\Lambda} /$, an Urdu voiceless unaspirated dental stop / $\mathbf{t} /$ is replaced with an English voiceless alveolar stop /t/ and an Urdu open back long vowel /a:/ is substituted with an English central weak vowel /ə/. Similarly, in the Urdu word khansama (sr. 132), an Urdu voiceless velar fricative $/ \chi /$ is changed with an English voiceless velar plosive $/ \mathbf{k} /$, an Urdu open back long vowel /a:/ is substituted with an English central weak vowel /o/ and an Urdu open, back nasalised long vowel /ãa:/ at the word ending position is replaced with an English open back long vowel /a:/.

iv. Substitution of a consonant, a vowel and deletion of a consonant: In this pattern an Urdu consonant is substituted with an English consonant, an Urdu vowel is substituted with an English vowel and an Urdu consonant often at the word final position is deleted in English. The Urdu words chador, shalwar, talwar and dharna (sr. 119, 124, 126, 129) are examples of such variation. In the word chador (sr. 119), an Urdu voiced aspirated dental stop /d/ is replaced with an English voiced alveolar plosive /d/, an Urdu back vowel/o:/ is changed with an English central vowel/o/ whereas an Urdu alveolar voiced trill $/ \mathbf{r} /$ at the syllable final position is deleted by English speakers. In the Urdu word shalwar (sr. 124), an Urdu retroflex voiceless fricative $/ \mathbf{J} /$ is substituted with an English labiodental voiceless fricative /f/, an Urdu central vowel/a/ is changed with an English central vowel $/ \mathbf{N} /$ and an Urdu alveolar voiced trill $/ \mathbf{r} /$ at the syllable final position is deleted. The same happens with the Urdu word talwar (sr. 126) in which an Urdu voiceless unaspirated dental stop $/ \mathbf{t} /$ is replaced with an English voiceless alveolar stop /t/, an Urdu central vowel /o/ is changed with an English central vowel / $\mathbf{\Lambda} /$ and an Urdu alveolar voiced trill $/ \mathbf{r} /$ at the syllable final position is omitted. In the Urdu word dharna (sr. 129), an Urdu voiced aspirated dental stop /d/ is replaced with an English voiced alveolar plosive /d/, an Urdu central vowel/o/ is changed with an English mind-central vowel /3:/ and an Urdu alveolar voiced trill /r/ at the syllable final position is deleted. Similarly, the Urdu words kurta and purdah (sr. 122, 123) also indicate substitution of a vowel, a consonant and the deletion of a consonant sound. In Urdu the word kurta (sr. 122), an Urdu back short vowel / $/$ / is substituted with an English mid-central vowel /3:/, an Urdu alveolar voiced trill /r/ at the syllable final position is omitted and an Urdu voiceless unaspirated dental stop / $\mathbf{t} /$ is replaced with an English voiceless alveolar stop /t/. In Urdu the word purdah (sr. 123), Urdu central vowel.

v. Similarly, the Urdu words messiah and sarkar (sr. 125, 127) also indicate substitution of a vowel, a consonant and omission of a consonant. In the Urdu word messiah (sr. 125), an Urdu front short vowel /I/ is replaced with an English triphthong/aiə/, whereas an Urdu glottal fricative /h/ and an Urdu central short vowel /ə/ are omitted. Similarly, in the word sarkar (sr. 127), an Urdu central short vowel /a/ is substituted with an English mid-central vowel /3:/, and an Urdu alveolar voiced trill /r/ at the coda positions 
of both syllable is deleted.

\subsection{Four Changes}

$2 \%$ words shown at serial 133-135 in table 4.1 from data show Levenshtein distance value 4 (four). These words indicate four phonemic changes in standard British English. Their description is as below:

i. Substitution of two vowels, a consonant and deletion of a consonant: the Urdu word mazdoor (Sr. 133) shows this variation. An Urdu central vowel /ə/ is replaced with an English central vowel $/ \mathbf{\Lambda} /$ and an Urdu voiced dental stop /d/ is substituted with an English voiced alveolar stop /d/. Moreover, an Urdu back long vowel /u:/ is substituted with an English diphthong /Uə/ and an Urdu alveolar voiced trill /r/ is omitted.

ii. Double substitution of a vowel, deletion of a consonant and substitution of a consonant: This variation can be found in the Urdu word cummerbund (sr. 134). An Urdu short vowel /o/ is substituted with an English central vowel / $\mathbf{A}$. An Urdu voiced alveolar trill $/ \mathbf{r} /$ is omitted whereas an Urdu voiced dental stop $/ \mathbf{d} /$ is substituted with an English voiced alveolar stop /d/.

iii. Substitution of two consonants and two vowels: The Urdu word tandoori (sr. 135) shows this variation. An Urdu voiceless dental stop /t $/$ is replaced with an English voiceless alveolar stop /t/. An Urdu short vowel /ə/ is replaced with an English short vowel/æ/. An Urdu voiced dental stop /d/ is substituted with an English voiced alveolar stop /d/ whereas an Urdu back short vowel/u/ is substituted with an English diphthong $/ \mathbf{U} /$.

\section{CONCLUSION}

In the light of data analysis, this study concludes that $16 \%$ Urdu loanwords into standard British English do not indicate any variation and retain their pronunciation whereas $84 \%$ words show phonemic changes in pronunciation. $42 \%$ words result in single phonemic change, $30 \%$ words have variation in two phonemes, $10 \%$ words have variation in three phonemes and $2 \%$ words result in variation of four phonemes. This phonemic variation employs different strategies like substitution, deletion and addition. Substitution is one the most prominent strategies. These phonemic changes seem to be systematic and form different patterns. Following these patterns, one can easily identify pronunciation of future Urdu loanwords into standard British English. 
International Journal of Innovation, Creativity and Change. www.ijicc.net

Volume 15, Issue 8, 2021

\section{REFERENCES}

Abdullah, A. B., \& Daffar, A. H. M. (2006). English loan words in the spoken Arabic of the southern part of Iraq: A sociolinguistic study. ADAB AL-BASRAH(41).

Al-Athwary, A. A. (2017). The Phonotactic Adaptation of English Loanwords in Arabic. Arab World English Journal (AWEJ) Volume, 8. doi:https://doi.org/10.24093/awej/vol8no3.25

Al-Qinal, J. B. (2002). Morphophonemics of loanwords in translation. Journal of King Saud University, 13, 1-132.

Atta, F., Syed, N. A., \& Bughio, F. A. (2017). A Phonological Treatment of Vowels in English Loanwords By Saraiki Speakers. ELF Annual Research Journal, 19, 43-62.

Bielenia-Grajewska, M. (2009). Linguistic borrowing in the English language of economics. Lexis. Journal in English Lexicology(3).

Bilal, A., Warraich, A., Fatima, N., Tiwana, S., \& Bhatti, R. (2012). Urdu loanwords in Pakistani English. Global Journal of Human Social Science Linguistics \& Education, $12(9)$.

Bukhari, S. (1985). Phonology of Urdu Language: Royal Book Company.

Bukhari, S. (1991). Urdu Zuban Ka Soti Nizaam aur Taqabli Mutalia (1st ed.). Islamabad, Pakistan: National Language Authority (National Language Promotion Department).

Chang, C. B. (2009). English loanword adaptation in Burmese. Journal of the Southeast Asian Linguistics Society, 1, 77-94.

Chohan, M. N., \& García, M. I. M. (2019). Phonemic Comparison of English and Punjabi. International Journal of English Linguistics, 9(4), 11. doi:https://doi.org/10.5539/ijel.v9n4p347

Crystal, D. (2008). A Dictionary of Linguistics and Phonetics (6th ed.). USA: Blackwell Publishing Ltd.

Dashti, F., \& Dashti, A. A. (2017). Morphological Adaptation of English Loanwords in Twitter: Educational Implications. International Journal of Higher Education, 6(3), 231-248. doi:https://doi.org/10.5430/ijhe.v6n3p231

Dictionary.com. (Ed.) (2020) Dictionary.com.

Garcia, M. I. M., \& Yapici, M. (2014). Common Vocabulary in Urdu and Turkish Language: A Case of Historical Onomasiology. Pakistan Vision, 15(1), 193.

Haqqi, S. u. H. (Ed.) فربنى تلفظ (2017). Islamabad: Muqtadra Qomi Zuban (National Language Authority).

Hasan, W., \& Khan, A. A. (2019). Phonological Analysis of English Loanwords in Dhani. International Journal of English Linguistics, 9(6).

Hornby, A. S., \& Cowie, A. P. (2020). Oxford Advanced Learners' Dictionary (10th ed.): Oxford University Press Oxford.

Hussain, Q., Mahmood, R., \& Mahmood, M. A. (2011). Vowel substitution: A comparative study of English loans in Punjabi and Urdu. International Journal of Linguistics, 3(1), 1-13. doi:https://doi.org/10.5296/ijl.v3i1.1022 
International Journal of Innovation, Creativity and Change. www.ijicc.net

Volume 15, Issue 8, 2021

Hussain, Q., Mahmood, R., \& Mahmood, M. A. (2012). Phonological Make-up of English Loanwords Incorporated into Punjabi via Urdu. Journal of Language Teaching \& Research, 3(5). doi:https://doi.org/10.4304/jltr.3.5.838-843

Kenstowicz, M., \& Suchato, A. (2006). Issues in loanword adaptation: A case study from Thai. Lingua, 116(7), 921-949. doi:https://doi.org/10.1016/j.lingua.2005.05.006

Khan, A. Q., \& Bukhari, N. H. (2011). Phonological Adaptation of English Loan Words in Pahari. Language in India, 11(1).

Khan, M. A. (1997). Urdu ka Soti Nizaam (1st ed.). Islamabad, Pakistan: National Language Authority (National Language Promotion Department).

Khan, N. W., Rizvi, S. Z., \& Farooq, M. (2020). The Study of English Loan Word Phonology in Urdu. International Journal of Psychosocial Rehabilitation, 24(9).

Levenshtein, V. (1966). Binary Codes Capable of Correcting Deletions, Insertions, and Reversals. Paper presented at the Soviet physics doklady.

Mahmood, R., Hussain, Q., \& Mahmood, M. A. (2011). Phonological Adaptations of English Words Borrowed into Punjabi. European Journal of Social Sciences, 22(2), 234-245.

Mangrio, R. A. (2016). The Morphology of Loanwords in Urdu: The Persian, Arabic and English Strands: Cambridge Scholars Publishing.

Miao, R. (2005). Loanword adaptation in Mandarin Chinese: Perceptual, phonological and sociolinguistic factors.

Narang, G. C. (2007). Urdu Zabaan aur Lisaaniyaat. Lahore, Pakistan: Sang-e-Meel Publications.

Population Census. (2017). Retrieved January 27, 2019 http://www.pbscensus.gov.pk/

Rafi, M. S. (2013). Urdu and English contact in an e-discourse: Changes and implications. Gomal university journal of research, 29(2), 78-86.

Roach, P. (2013). English Phonetics and Phonology: A practical course (fourth ed.). New Delhi, India: Cambridge University Press.

Saleem, A. M., Kabir, H., Riaz, M. K., Rafique, M. M., Khalid, N., \& Shahid, S. R. (2002). Urdu consonantal and vocalic sounds. CRULP Annual Student Report.

Sanders, N. C., \& Chin, S. B. (2009). Phonological Distance Measures. Journal of Quantitative Linguistics, 16(1), 96-114.

Simons, G. F., \& Fennig, C. D. (2018). Ethnologue: Languages of the world. SIL International. Retrieved from https://www.ethnologue.com/country/PK

Sipra, M. (2013). A Linguistic Study of Borrowings from English to Urdu. International Journal of Applied Linguistics \& English Literature, 2(1). doi:https://doi.org/10.7575/ijalel.v.2n.1p.203

Trudgill, P. (1992). Introducing Language and Society. England: Penguin Books Ltd.

Urdu Lughat. (2017) (online ed.). Karachi: Urdu Dictionary Board.

Usman, M., Ali, S. F., \& Masood, A. (2003). Syllabification of English words when spoken in Urdu. Akhbar-e-Urdu. National Language Authority, Islamabad, Pakistan.

Vendelin, I., \& Peperkamp, S. (2006). The influence of orthography on loanword adaptations. Lingua, 116(7), 996-1007. doi:https://doi.org/10.1016/j.lingua.2005.07.005 